\title{
Performance of optimised prosthetic ankle designs that are based on a hydraulic variable displacement actuator (VDA)
}

\author{
James Gardiner, Abu Zeeshan Bari, Laurence Kenney, Martin Twiste, David Moser, Saeed Zahedi and \\ David Howard
}

\begin{abstract}
Current energy storage and return (ESR) prosthetic feet only marginally reduce the cost of amputee locomotion compared to basic solid ankle cushioned heel (SACH) feet, possibly due to their lack of push-off at the end of stance. To our knowledge, a prosthetic ankle that utilises a hydraulic variable displacement actuator (VDA) to improve push-off performance has not previously been proposed. Therefore, here we report a design optimisation and simulation feasibility study for a VDA based prosthetic ankle. The proposed device stores the eccentric ankle work done from heel strike to maximum dorsiflexion in a hydraulic accumulator and then returns the stored energy to power push-off. Optimisation was used to establish the best spring characteristic and gear ratio between ankle and VDA. The corresponding simulations show that, in level walking, normal push-off is achieved and, per gait cycle, the energy stored in the accumulator increases by $22 \%$ of the requirements for normal push-off. Although the results are promising, there are many unanswered questions and, for this approach to be a success, a new miniature, low-losses, lightweight VDA would be required that is half the size of the smallest commercially available device.
\end{abstract}

Index Terms-prosthetics, ankle, hydraulics, biomechanics, engineering

\section{INTRODUCTION}

$\mathrm{T}$ HE cost of locomotion (joules per $\mathrm{kg}$ per metre) is significantly higher for lower limb amputees than for their healthy counterparts [1]-[4], affecting their mobility and quality of life. Furthermore, current energy storage and return (ESR) prosthetic feet only marginally reduce the cost of

James Gardiner was with CSE, University of Salford, Salford, M5 4WT, UK. He has moved to IACD, University of Liverpool, Liverpool, L7 8TX, UK (e-mail: j.d.gardiner@liverpool.ac.uk)

Abu Zeeshan Bari was with the School of Health Sciences, University of Salford, Salford, M5 4WT, UK. He has moved to NED University of Engineering \& Technology, Karachi, Pakistan (e-mail: bari@neduet.edu.pk)

Laurence Kenney is with the School of Health Sciences, University of Salford, Salford, M5 4WT, UK (e-mail: L.P.J.Kenney@salford.ac.uk)

Martin Twiste is with the School of Health Sciences, University of Salford, Salford, M5 4WT, UK (e-mail: M.Twiste@salford.ac.uk)

David Moser is with Blatchford Products Ltd., Basingstoke, Hampshire, RG24 8PZ, UK (email: David.Moser@blatchford.co.uk)

Saeed Zahedi is with Blatchford Products Ltd., Basingstoke, Hampshire, RG24 8PZ, UK (email: Saeed.Zahedi@blatchford.co.uk)

David Howard is with the CSE, University of Salford, Salford, M5 4WT, UK (e-mail: D.Howard@salford.ac.uk) amputee locomotion compared to basic solid ankle cushioned heel $(\mathrm{SACH})$ feet [5], [6], possibly as a result of their lack of push-off power at the end of stance. Studies of healthy walking suggest that push-off saves energy by either powering the leg into swing [7], propelling the body, helping to reduce contralateral collision losses at heel strike [8], [9], or some combination of these. Regardless of its function, push-off is clearly important and missing on the prosthetic side during amputee gait [10]-[12]. ESR feet are unlikely to push-off effectively due to their inability to produce a plantar-flexion moment when in a plantar-flexed position. Indeed, the neutral (zero moment) ankle angle of a passive prosthesis corresponds to normal standing; therefore, once the prosthesis moves into plantar-flexion it produces a dorsi-flexion moment, the opposite of what is required at push-off. As a result, amputees have to compensate for the lack of ankle push-off power using more proximal joints and their sound limb. Indeed, our analysis of power flows in gait [13] indicates that, at push-off, in healthy subjects power flows proximally from the ankle, whereas in amputees power flows distally from the hip.

With the aim of improving on current ESR feet, several research teams have developed prototype prosthetic feet that are designed to restore more natural motion including the missing push-off at the end of stance. Williams et al. [14] describe a prototype prosthetic foot which engages a clutch at foot flat so that an 'Achilles' spring is stretched through midstance to maximum dorsiflexion (storing energy) and then aids push-off during late-stance plantarflexion (releasing energy). At the end of stance when load is removed, the clutch is released resetting the system. Although the aim was to achieve slope adaptation, their design is equally applicable to effective energy storage and return. Similarly, Collins and Kuo [15] designed a prototype prosthetic foot which engages a clutch to lock in the strain energy stored in a spring during load acceptance. The clutch is disengaged at the end of stance and the stored strain energy released to aid push-off. Whilst their prototype showed an increase in peak push-off power, compared to more conventional prosthetic feet, it failed to improve amputee walking efficiency [16] perhaps because the eccentric work done in load acceptance is relatively small. Both of these designs use clutch and spring mechanisms to store energy, consequently there is no control over the rate at which energy is returned. The BiOM foot and ankle developed 
NOMENCLATURE

\begin{tabular}{|c|c|c|}
\hline Symbol & Definition & Value \\
\hline$A$ & $\begin{array}{l}\text { spring model parameter } \\
\text { (selected by optimiser) }\end{array}$ & \\
\hline$B$ & $\begin{array}{l}\text { spring model parameter } \\
\text { (selected by optimiser) }\end{array}$ & \\
\hline$B_{m}$ & hydraulic oil bulk modulus & $1.66 \mathrm{e} 9 \mathrm{~N} / \mathrm{m}^{2}$ \\
\hline$C_{f}$ & VDA coulomb friction coefficient & \\
\hline$C_{s}$ & VDA slip coefficient & \\
\hline$C_{v}$ & VDA viscous friction coefficient & \\
\hline$D$ & maximum VDA displacement & \\
\hline$I$ & $\begin{array}{c}\text { combined moment of inertia of gearbox and } \\
\text { VDA }\end{array}$ & \\
\hline$k$ & coefficient in gearbox efficiency function & \\
\hline$k_{h}$ & specific heat ratio & 1.4 \\
\hline$P$ & hydraulic system pressure & \\
\hline$P_{p}$ & accumulator pre-charge pressure & 100 bar \\
\hline$Q$ & VDA flow rate & \\
\hline$Q_{l}$ & VDA flow losses & \\
\hline$R$ & $\begin{array}{c}\text { gearbox ratio } \\
\text { (selected by optimiser) }\end{array}$ & \\
\hline$T$ & VDA torque & \\
\hline$T_{f}$ & VDA friction torque & \\
\hline$T_{g b f}$ & gearbox friction torque & \\
\hline$T_{\text {in }}$ & gearbox input torque & \\
\hline$T_{\text {out }}$ & gearbox output torque & \\
\hline$T_{\max }$ & gearbox maximum torque & \\
\hline$T_{s}$ & spring torque & \\
\hline$V$ & current accumulator oil volume & \\
\hline$V_{a}$ & $\begin{array}{l}\text { accumulator capacity } \\
\text { (i.e. maximum oil volume) }\end{array}$ & $2.5 \mathrm{e}-04 \mathrm{~m}^{3}$ \\
\hline VDA & variable displacement actuator & \\
\hline$V_{\min }$ & accumulator minimum oil volume & $1.0 \mathrm{e}-6 \mathrm{~m}^{3}$ \\
\hline$V_{r}$ & $\begin{array}{l}\text { VDA volume ratio } \\
\text { (clearance volume at piston top dead } \\
\text { centre/swept volume) }\end{array}$ & 0.1 \\
\hline$x$ & $\begin{array}{l}\text { fractional VDA displacement } \\
\quad \text { (varies between } 0 \text { and } 1)\end{array}$ & \\
\hline$\alpha$ & VDA angular acceleration & \\
\hline$\lambda$ & $\begin{array}{l}\text { spring model parameter } \\
\text { (selected by optimiser) }\end{array}$ & \\
\hline$\eta$ & gearbox efficiency & \\
\hline$\theta_{a}$ & ankle angle & \\
\hline$\mu$ & hydraulic oil viscosity & $0.017 \mathrm{Ns} / \mathrm{m}^{2}$ \\
\hline$\omega$ & VDA rotational speed & \\
\hline$\omega_{\text {in }}$ & gearbox input speed & \\
\hline$\omega_{\text {out }}$ & gearbox output speed & \\
\hline
\end{tabular}

by Hugh Herr's team at MIT [17], [18] uses a combination of springs, electric motor and battery to improve amputee walking performance. Their design reduces the cost of locomotion to the point where, under laboratory conditions, trans-tibial amputees using the device are not statistically significantly different from healthy controls [19]. However, their device is primarily active requiring electrical power to improve push-off and hence walking efficiency. The design also includes elastic energy storage and return components which, because they are similar to those seen in commercial ESR prostheses, are unlikely to contribute to improved pushoff. Indeed, the reduction in metabolic cost for the amputee is slightly less than the metabolic equivalent of the energy input from the battery [19], implying that there is no improvement in elastic energy storage and return over commercial ESR prostheses. Therefore, the BiOM doesn't fully utilise the negative ankle work done in early and mid-stance, which if stored effectively should be able to power, if not all, at least a significant proportion of push-off.

Hydraulic actuation of prosthetic limbs has been suggested before [20]-[23] and many commercial prosthetic knees utilise hydraulic damping. However, to our knowledge, the use of a hydraulic variable displacement actuator (VDA) within a prosthesis has not previously been proposed. In this context, candidates for a suitable VDA may be a miniature axial or radial piston pump, where displacement can be adjusted by varying swash plate angle or stator position respectively. A major advantage of VDAs, over other hydraulic actuators, is that their torque output can be continuously adjusted by changing their displacement, which makes a natural ankle torque profile achievable. Their disadvantages are: a) their relatively high flow and friction losses; and b) their mechanical complexity. Hydraulic designs have unique advantages for prosthetics applications. Firstly, their high operating pressures (up to 200bar) mean they have very high power densities and therefore are well suited to miniaturisation. Secondly, hydraulic accumulators are well suited to rapid energy storage and return over very large numbers of repeated cycles, which is not the case for batteries. Finally, hydraulic actuation is ideally suited for transferring energy between joints because the transfer mechanism involves only pipes and fluid, rather than gears and linkages. This ease of energy transfer is of particular importance for higher level amputees who could benefit if, for example, the excess of eccentric work at the knee could be stored and used in a controlled manner at the ankle.

Here we report on a design optimisation and simulation study to establish the feasibility of a VDA and accumulator based prosthetic ankle and to assess whether its advantages outweigh its disadvantages.

\section{METHODS}

\section{A. Model Overview}

In the proposed design (Fig. 1) the ankle is driven by a VDA via a reduction gearbox, which is included so that, using optimisation, we can establish the best trade-off between gearbox and VDA losses. Increasing the gearbox ratio reduces the size of the VDA and the associated losses but increases the gearbox friction losses. A spring acts in parallel to the VDA and gearbox, reducing the torque/power they need to provide and thus making them smaller, lighter and more efficient. 


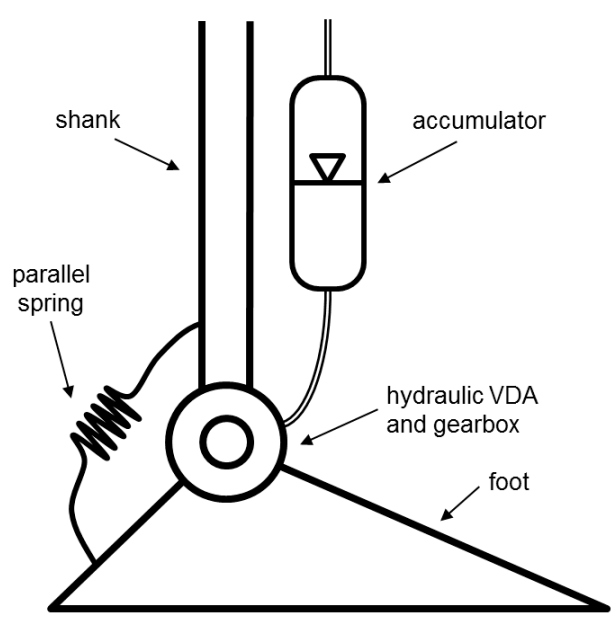

Fig. 1. Schematic of the proposed VDA based prosthetic ankle. The ankle is driven by a hydraulic variable displacement actuator (VDA) through a gearbox. The parallel spring is connected between the foot and shank of the prosthesis and serves to reduce the torque required from the hydraulic VDA and gearbox. An accumulator is used to store and return energy.

Depending on whether it is doing negative or positive work, the VDA either pumps oil into the accumulator (storing energy) or it acts as a motor taking oil from the accumulator (returning energy). With no parallel spring, the VDA will be in pump mode during early and mid-stance (eccentric ankle work) and in motor mode during push-off (concentric ankle work). However, as a result of design optimisation, the parallel spring can modify the shape of the torque curve required from the VDA and gearbox in an unexpected manner such that the VDA may be pumping when it would have been motoring in a design without a spring. We assume that there is no delay in response to changes of ankle torque demand (i.e. instantaneous VDA control). In reality, some delays would occur but this assumption was considered appropriate for the purposes of assessing the design's mechanical feasibility. Design optimisation and simulation were performed using custom simulation code written in MATLAB ${ }^{\circledR}$ R2014b and by utilising the optimisation toolbox (The MathWorks, Inc., Natick, Massachusetts, United States)

\section{B. Input data}

The ultimate aim of prosthetic leg design is to allow an amputee to walk 'naturally'. In reality, most amputees walk with an asymmetric and impaired gait (even with state-of-theart prosthetic legs), which tends to worsen as the seriousness of the amputation increases [4], [24], [25]. Nevertheless, for simulation purposes we use ankle angle and torque profiles for healthy walking since this is the ultimate aim of prosthetic leg design. Furthermore, theoretical prediction of amputee gait with an as yet untested prosthesis design is incredibly difficult since the actual ankle angle profile will be determined by the amputee's impairments and reaction to the device, which can only be studied through physical prototyping and in-vivo testing.

We chose to use optimistic data, in terms of the amount of eccentric work available for storing and returning, because there is an argument that amputees would benefit from walking in a manner that provides good push-off if it helps to reduce their overall cost of locomotion. The simulation input data (Fig. 2) were taken from Bari (2013) [26] and are for healthy level walking at self-selected speed. It is clear from the area under the power plot (Fig. 2) that the work input to the hydraulic ankle (i.e. negative or eccentric work at the ankle) is quite large and there is a significant amount of energy available to be stored and then returned for push-off (approx. 21J up to the start of push-off). Although this input data is optimistic with respect to the amount of eccentric work done, it is nevertheless collected from healthy gait and is similar to other published studies [11], [12], [27]. The data has been smoothed (essential when using numerical differentiation) and also made periodic to ensure the system returns to its starting position. If the data is not periodic, design optimisation can falsely take advantage of small differences in strain and kinetic energy between the start and end of the gait cycle, which are not available in periodic walking.
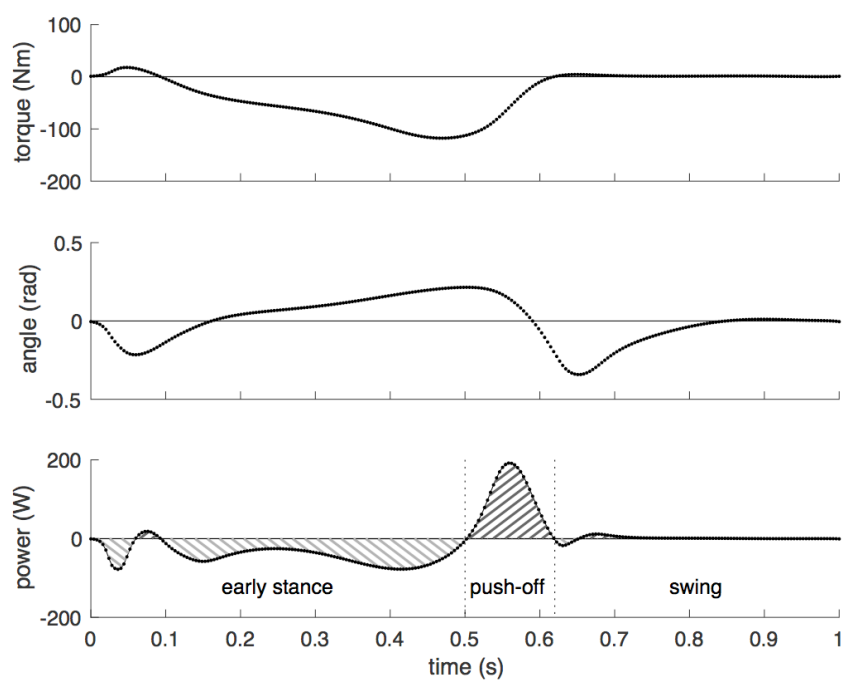

Fig. 2. Input ankle data for level walking at self-selected speed for one complete gait cycle including torque (top), angle (middle) and power (bottom). The light hatched area under the power curve shows the eccentric work that the ankle does prior to push-off, which is available to be stored in the spring and accumulator. The dark hatched area shows the work that needs to be returned to provide push-off (typically missing in amputee gait data).

\section{Spring Model}

The spring acts in parallel to the VDA and gearbox, reducing the torque/power they need to provide and thus making them smaller, lighter and more efficient. Hence, we adopted a spring curve that closely matches the ankle torque versus ankle angle profile of healthy gait (Fig. 3), a curve sometimes referred to as the 'pseudo stiffness' of the ankle joint, using the following exponential function

$$
T_{s}=A e^{\lambda \theta_{a}}+B
$$

The function can be forced to pass through the origin (by setting $A=-B$ ), so that at zero ankle angle there is no torque 


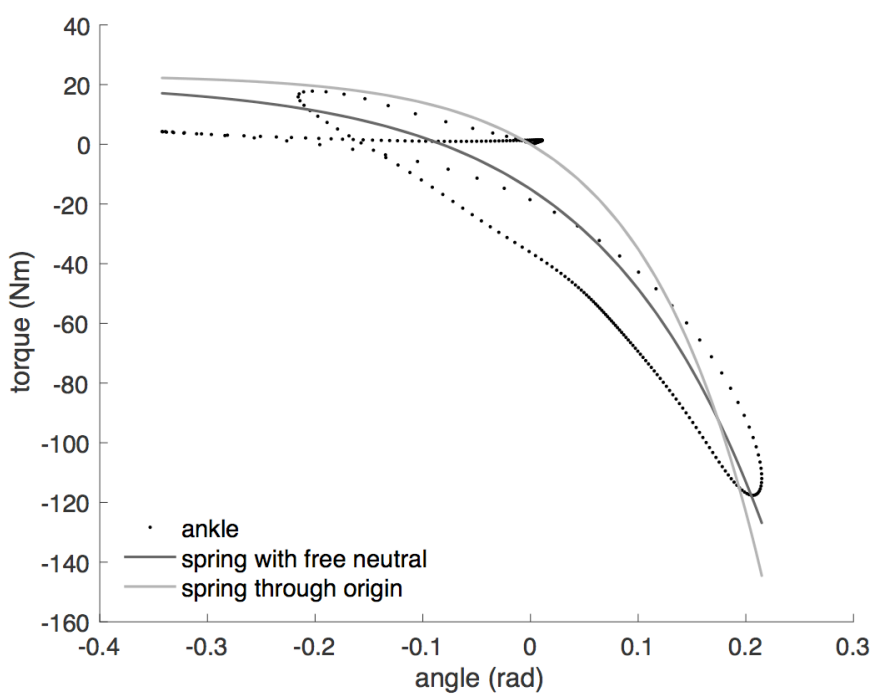

Fig. 3. Ankle torque versus ankle angle during healthy level walking (dots). Two example of the exponential spring characteristic $T_{s}=A e^{\lambda \theta_{a}}+B$ are shown (free neutral (dark grey) and through the origin (light grey)), which closely match the healthy ankle torque versus ankle angle profile.

output (i.e. when standing upright there is no spring torque). Alternatively, $A$ and $B$ can be independent so that the spring curve is free to move away from the origin depending on the parameters selected by the optimisation software. The spring is assumed to be $100 \%$ efficient, which is reasonable since any spring losses that do occur will be insignificant compared to the losses in the gearbox and VDA.

Our spring model has a non-linear load-displacement relationship which, in practice, would require a custom spring to be manufactured. Typically, non-linearity in spring behavior is achieved by variations in spring geometry, rather than material properties. For example, both a tapered spring with constant pitch and a straight spring with variable pitch will exhibit non-linear load-displacement relationships. If complicated non-linear behaviors are desired then more complex variations in geometry are required. Jutte and Kota [28], [29] present a general non-linear spring synthesis methodology which produces beam spring geometries applicable to any prescribed non-linear load-displacement function.

\section{Gearbox model}

The output speed of the gearbox follows the simple relationship

$$
\omega_{\text {out }}=\omega_{\text {in }} R
$$

The output torque is given by the following relationship, which includes gearbox friction torque

$$
T_{\text {out }}=\left(\frac{T_{\text {in }}-\operatorname{sign}\left(\omega_{\text {in }}\right) T_{\text {gbf }}}{R}\right)
$$

The sign of the input angular velocity is used to ensure that the friction torque always opposes the direction of motion. The gearbox also has inertia, which is based upon a curve fit of a manufacturer's data and lumped together with that of the VDA (hence not in the equation above). The inertial term $(I \alpha)$ in equation (9) turned out to be insignificant and, hence, the calculation of inertia is not reported in detail.

The gearbox friction torque is based on a manufacturer's quoted efficiencies (assumed to be reliable) and the maximum absolute torque $\left(T_{\max }\right)$ experienced by the gearbox over the gait cycle (the latter representing gearbox size). Hence gearbox friction torque is given by

$$
T_{g b f}=T_{\max } \frac{(100-\eta)}{100}
$$

where

$$
T_{\max }=\max \left|T_{i n}\right|
$$

Generally, gearbox efficiency decreases with ratio, and the following empirical function was fitted to the manufacturer's data using a least squares approach to determine the exponent $k$. The mean error between equation (6) and the experimental data is $0.60 \%$.

$$
\eta=100-(R-1)^{k}
$$

The function is forced through the point where a gearbox ratio of 1 provides $100 \%$ efficiency, so that a model with a ratio of 1 is identical to a model without a gearbox.

\section{E. VDA model}

Our VDA model is based on the model and experimental data presented by McCandlish and Dorey [30] for a radial piston pump . However, in our extended version of the model, to improve the fit to the original experimental data, the three empirical coefficients $\left(C_{s}, C_{V}\right.$ and $\left.C_{f}\right)$ vary as linear functions of both speed and pressure and also a correction is applied to the friction torque to obtain a better fit to the experimental data when the VDA operates at a fractional displacement $x$ of less than 1 (the maximum). We assume that the data presented in McCandlish and Dorey [30] is representative of the performance of the VDA in our design, even though their data was gathered under steady state conditions whereas in our application the VDA speed and direction of rotation vary rapidly. However, no better data was available in the literature and the only alternative to an empirical model would have been a detailed model of the VDA's internal fluid mechanics, which was considered far too complex a solution for our feasibility study. The VDA flow rate is modelled as follows

$$
Q=x \omega D-Q_{l}
$$

with the flow losses $Q_{l}$ given by

$$
Q_{l}=C_{s}\left(\frac{P D}{\mu}\right)+\frac{P|\omega| D}{B_{m}}\left(V_{r}+\frac{1+x}{2}\right)
$$


The first term models the leakage losses of the VDA and the second term the compressibility losses. The torque model from McCandlish and Dorey [30] is as follows

$$
T=x P D+\operatorname{sign}(\omega) T_{f}+I \alpha
$$

where mechanical friction torque is given by

$$
T_{f}=C_{v}(\mu|\omega| D)+C_{f}(P D)
$$

The two terms model viscous friction and coulomb friction respectively. The three empirical coefficients $\left(C_{s}, C_{V}\right.$ and $\left.C_{f}\right)$ in the equations above are assumed to vary with both speed and pressure in a linear fashion as follows

$$
C=a|\omega|+b P+k
$$

where $a, b$ and $k$ are constants determined through a least squares fit of equations (8), (10) and (11) to the experimental radial piston pump data presented in McCandlish and Dorey [30]. This is an extension to the approach adopted by McCandlish and Dorey [30].

The data presented in McCandlish and Dorey [30] shows that the friction torque $\left(T_{f}\right)$ is reduced when the VDA operates at a fractional displacement $x$ of less than 1, whereas the flow loss is mostly unaffected. This is not taken into account in equation (10) and, therefore, we introduced an additional correction that depends on the fractional displacement $x$. Inspection of the experimental data suggested a linear relationship between friction torque and fractional displacement and the following least squares fit was obtained

$$
T_{f(\text { corrected })}=T_{f}(0.4983|x|+0.5017)
$$

At a fractional displacement of $x=0.25$, the mean error in friction torque between our extended VDA model and the experimental data in McCandlish and Dorey [30] is reduced from 35.0 to $11.2 \%$ as a result of incorporating this correction.

The VDA inertia was estimated using an equation based on dimensional data for the rotating parts of typical axial piston pumps obtained from manufacturers' catalogues. The VDA inertia was combined with the gearbox inertia to give the total inertia I in equation (9). The inertial term $(I \alpha)$ in equation (9) turned out to be insignificant and, hence, the calculation of inertia is not reported in detail.

\section{F. Accumulator model}

We assumed that a gas-charged diaphragm accumulator would be most appropriate in this application because it is well suited to miniaturisation and lighter than other designs. The accumulator acts in a similar manner to a battery, storing and returning energy as required by the VDA depending on whether it is pumping or motoring. It also determines the system pressure. The accumulator is assumed to be lossless because it charges and discharges quickly (i.e. over one gait cycle) and the pressure/volume/temperature changes are very small, meaning that it is reasonable to assume adiabatic operation. Furthermore, the very small volume changes mean that any change in diaphragm shape is very small.

The VDA flow rate is integrated over each simulation time step to either fill or empty the accumulator, depending on whether the VDA is pumping or motoring, which determines the instantaneous oil volume $V$. Assuming polytropic expansion of the gas in the accumulator, Bari [26] showed that the system pressure is then given by

$$
P=P_{p}\left(\frac{V_{a}-V_{\min }}{V_{a}-V}\right)^{k_{h}}
$$

The initial oil volume of the accumulator is set so that the system pressure is 200 bar at the start of each simulation, which is a typical operating pressure for hydraulic systems.

\section{G. Simulation implementation}

The mathematical model described above was used as the basis for creating custom simulation code, written in MATLAB $^{\circledR} \quad$ R2014b (The MathWorks, Inc., Natick, Massachusetts, United States). This was written in the form of a function with design parameters (to be optimised) as the input arguments and the increase in accumulator pressure over the gait cycle as the output. In this context, the accumulator pressure increase is used as the optimisation objective that we wish to maximise. This is equivalent to maximising the energy stored in the accumulator, which in turn corresponds to minimising energy losses.

The simulation function is called iteratively by the chosen optimisation function from the MATLAB optimisation toolbox (The MathWorks, Inc., Natick, Massachusetts, United States), which alters the design parameters (function inputs) to find an optimal design which maximises the increase in accumulator pressure over the gait cycle (function output). The following section describes in detail the way in which design optimisation was undertaken.

\section{H. Optimisation}

Design optimisation was undertaken using the fminsearch function in the MATLAB optimisation toolbox, which finds the minimum of an unconstrained multivariable function. This was used to find the best set of independent design variables (gear box ratio $R$ and spring coefficients $A, B$ and $\lambda$ ), which maximise the energy stored in the accumulator over the gait cycle (i.e. maximise the increase in accumulator pressure). This is equivalent to minimising the losses, which in essence the optimiser achieves by finding a spring characteristic that reduces the torque required from the actuator (gearbox and VDA) and finding a gearbox ratio that gives a good trade-off between gearbox losses and VDA losses. Furthermore, every time the optimiser changes the independent design variables (gear box ratio $R$ and spring coefficients $A, B$ and $\lambda$ ), the dependent design parameters, including various model coefficients, are recalculated. In particular the VDA 
displacement $D$ is recalculated for every optimiser run so that the maximum fractional displacement, $\max (x)$, remains close to 1 , which is necessary to minimise its size without allowing it to be undersized. In order to achieve this, at the start of each iteration we assume that the $\max (x)$ will be equal to 0.95 , which in turn allows us to calculate a displacement for the VDA and run the simulation. In reality, due to the nature of the model, the final $\max (x)$ at the end of each iteration is typically larger than 0.95 at around $0.96-0.97$. This is important since, if $\max (x)>1$, then the VDA is undersized and cannot produce the necessary torque. Conversely, if $\max (x)$ is significantly less than 1, the VDA will be oversized and less efficient. In our simulation, therefore, the VDA is always fractionally oversized by a few percent compared to a 'perfect' solution, however this ensures that it can provide the torque required for the full gait cycle.

In this feasibility study we considered three alternative designs, each of which was optimised. These alternatives correspond to the following optimisation problems

- DESIGN_1-Optimise gearbox ratio $R$ and spring parameters $A, B$ and $\lambda$, which corresponds to a spring with a free neutral point (i.e. not forced through the origin of Fig. 3)

- DESIGN_2-Optimise gearbox ratio $R$ and spring parameters $A$ and $\lambda$, with $B=-A$, which corresponds to a spring that is forced to go through the origin of Fig. 3 (i.e. when standing upright there is no spring torque)

- DESIGN_3 - Optimise gearbox ratio $R$ only for a system with no parallel spring

To ensure that the optimiser had found global solutions rather than local maxima, the optimiser was started from different initial conditions (values of $R, A, B$ and $\lambda$ ) and we checked that the same solution was repeatedly being found. Additionally, by searching either side of the optimum value for each variable, we checked to ensure that a true maxima has been found rather than a local maxima.

\section{RESULTS}

The optimiser found consistent solutions (Table 1) for all three design cases (i.e. starting from different initial values for the independent design variables). These three designs were selected by the optimiser to maximise the energy stored in the accumulator over the gait cycle. In all three cases, the gearbox ratios are very similar ranging from 25.97 in DESIGN_1 to 27.24 in DESIGN_3, which probably confirms the idea that the optimiser finds a gearbox ratio that gives a good trade-off between gearbox losses and VDA losses, a problem that is common to all three designs. However, the spring characteristics differ significantly between DESIGN_1 and DESIGN_2 (DESIGN_3 has no spring). DESIGN_2 has a fixed neutral point at the origin (i.e. when standing upright there is no spring torque), whereas DESIGN_1 has a free neutral point selected by the optimiser.

Differences in the optimised (independent) design variables
TABLE I

BEST SOLUTIONS FOUND BY THE OPTIMISER FOR THE INDEPENDENT DESIGN VARIABLES

\begin{tabular}{cccc}
\hline \hline $\begin{array}{c}\text { Optimised } \\
\text { design variable }\end{array}$ & $\begin{array}{c}\text { DESIGN_1 } \\
\text { free neutral } \\
(\mathrm{R}, \mathrm{A}, \mathrm{B} \text { and } \lambda)\end{array}$ & $\begin{array}{c}\text { DESIGN_2 } \\
\text { fixed neutral } \\
(\mathrm{R}, \mathrm{A}=-\mathrm{B}, \text { and } \lambda)\end{array}$ & $\begin{array}{c}\text { DESIGN_3 } \\
\text { no spring } \\
\text { (only R) }\end{array}$ \\
\hline Gearbox ratio & 25.9705 & 26.6749 & 27.2445 \\
Spring A & 35.8650 & 23.2248 & - \\
Spring lambda & 6.5900 & 9.2061 & - \\
Spring B & -20.8522 & -23.2248 & - \\
\hline \hline
\end{tabular}

TABLE II

RELATIVE PERFORMANCE OF THREE ALTERNATIVE DESIGNS, SHOWING KEY DEPENDENT DESIGN VARIABLES (SIZES OF THE GEARBOX AND VDA) AND INCREASES IN STORED ENERGY IN ACCUMULATOR

\begin{tabular}{cccc}
\hline \hline $\begin{array}{c}\text { Dependent } \\
\text { design } \\
\text { variable }\end{array}$ & $\begin{array}{c}\text { DESIGN_1 } \\
\text { free neutral } \\
(\mathrm{R}, \mathrm{A}, \mathrm{B} \text { and } \lambda)\end{array}$ & $\begin{array}{c}\text { DESIGN_2 } \\
\text { fixed neutral } \\
(\mathrm{R}, \mathrm{A}=-\mathrm{B} \text {, and } \lambda)\end{array}$ & $\begin{array}{c}\text { DESIGN_3 } \\
\text { no spring } \\
\text { (only R) }\end{array}$ \\
\hline $\begin{array}{c}\text { max VDA } \\
\text { displ. (D) }\end{array}$ & $256.1 \mathrm{~mm} 3 / \mathrm{rev}$ & $427.0 \mathrm{~mm} 3 / \mathrm{rev}$ & $1416.0 \mathrm{~mm} 3 / \mathrm{rev}$ \\
$\begin{array}{c}\text { max gearbox } \\
\text { torque } \\
\text { max VDA } \\
\text { torque }\end{array}$ & $21.2107 \mathrm{Nm}$ & $36.3579 \mathrm{Nm}$ & $117.61 \mathrm{Nm}$ \\
$\begin{array}{c}\text { increase in } \\
\text { acc. energy }\end{array}$ & $\begin{array}{c}+22 \% \text { of } \\
\text { normal push-off }\end{array}$ & $\begin{array}{c}-11 \% \text { of } \\
\text { normal push-off }\end{array}$ & $\begin{array}{c}-176 \% \text { of } \\
\text { normal push-off }\end{array}$ \\
\hline \hline
\end{tabular}

result in corresponding differences in the key dependent design variables that determine the physical sizes of the VDA and gearbox (Table 2). Notably, there are significant differences in the maximum VDA displacement and the maximum gearbox torque for the three designs, with DESIGN_2 and DESIGN_3 requiring components that are approximately 1.7 and 5.5 times larger than DESIGN_1 respectively.

Fig. 4 shows how torque and power vary over the gait cycle for DESIGN_1 and DESIGN_2. The designs produce the ankle input data (solid curves) that represent a normal gait with full push-off. The dashed and dash-dot curves show how the torque and power are divided between the actuator (gearbox and VDA) and the parallel spring. In both cases, it is clear that the optimiser has selected the spring characteristic to minimise the maximum actuator torque magnitude, and hence minimise actuator size and losses. It achieves this by evening out the actuator torque requirement over the gait cycle (i.e. by achieving max + ve torque $\approx \mid$ min - ve torque $\mid)$. This leads to some rather counter intuitive effects such as the actuator opposing the spring during push-off and also during swing for DESIGN_1.

The most notable difference between the two cases is in swing, which was expected as DESIGN_2 has a more typical spring neutral point (zero torque at zero angle), which also means the actuator torque approaches zero during swing. The DESIGN_1 results are particularly interesting as the optimiser has taken advantage of the free neutral point to increase the energy stored over the gait cycle and this is achieved despite the actuator working against the spring during swing. 

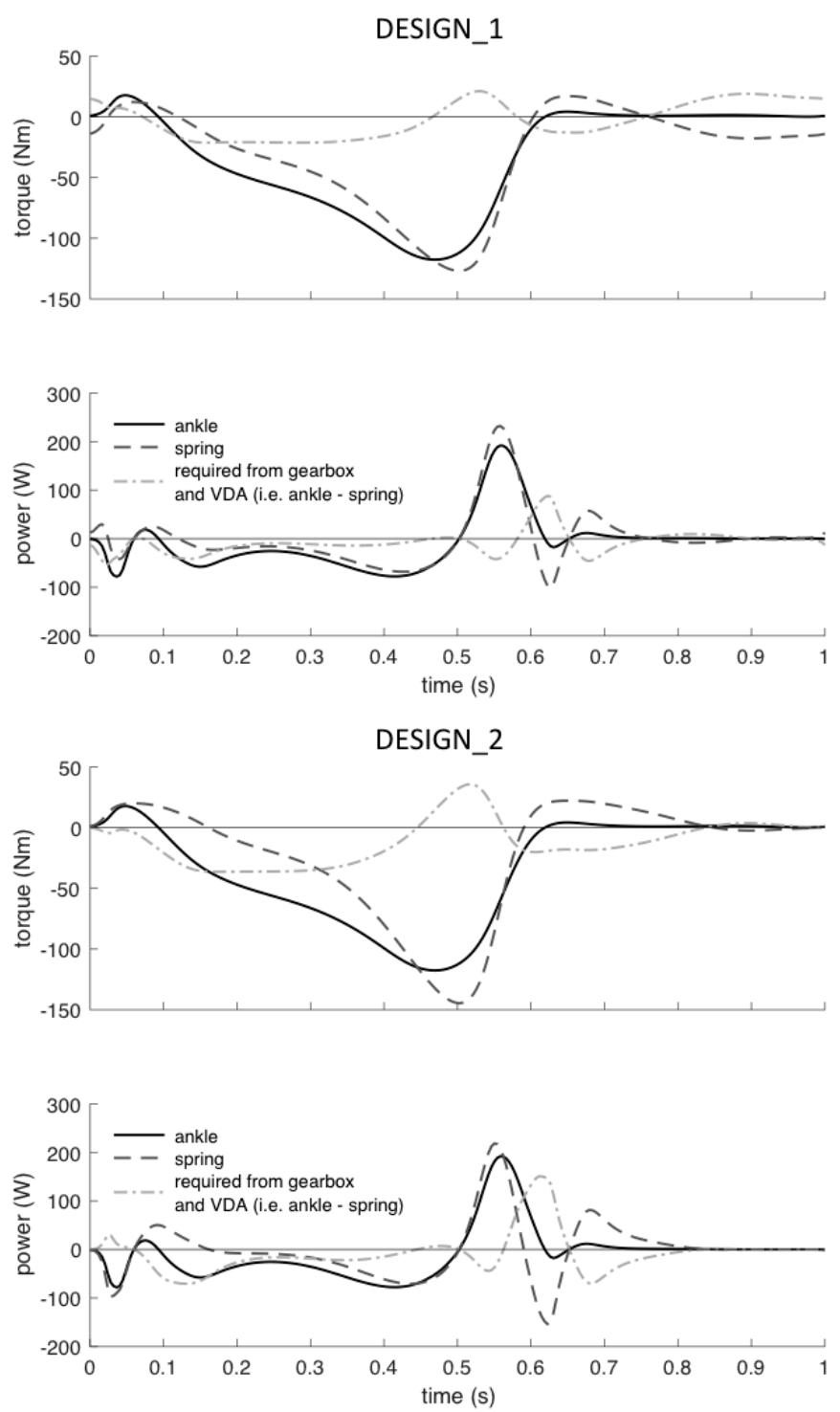

Fig. 4. Torque and power over the gait cycle for ankle, spring and actuator (gearbox and VDA) for DESIGN_1 - free neutral (top) and DESIGN_2 fixed neutral (bottom). The instantaneous ankle torque is the sum of the spring and actuator torques.

Table 2 summarises the relative performance of the three optimised designs, which is reflected in the net energy increase over the gait cycle, this being what the optimiser was trying to maximise (equivalent to minimising losses). The net energy increase is shown as a percentage of that required for normal push-off (12.24 joules). It is quite clear that DESIGN 1 (free neutral) is superior to the other 2 designs and sufficiently so for us to focus on DESIGN_1 in the remainder of this paper.

\section{DISCUSSION}

DESIGN_1 outperforms the other 2 designs (Table 2) despite the fact that, because of the spring's free neutral point, the actuator (gearbox and VDA) works against the parallel spring during swing (Fig. 4). Indeed, the use of a parallel spring is clearly essential to reduce the demand on the actuator (gearbox and VDA) if the sizes of the gearbox and VDA are to be minimised along with the associated losses. This is reflected in the maximum values of VDA displacement, VDA torque, and gearbox torque shown in Table 2. Overall the simulated performance of DESIGN_1 is promising and suggests that further research may yield rewards. However, the success of any future device will depend on a number of factors related to energy efficiency and practical design issues, which are discussed below.

\section{A. Energy efficiency}

In level walking, over each gait cycle Design_1 increases the energy stored in the accumulator by $+22 \%$ of what is required for normal push-off. Therefore, in theory continuous level walking without energy loss is feasible with Design_1 since the accumulator will continue to gain pressure until its capacity is reached. However, real world walking is rarely continuously level and therefore periods of uphill and downhill walking are to be expected. In particular, uphill walking is likely to result in a loss of accumulator pressure because concentric work exceeds eccentric work. However, due to Design_1's ability to re-pressurise the accumulator during level walking, short periods of uphill walking may be compensated for by longer periods of level walking. To put this in context, preliminary work suggests that 6 gait cycles of level walking would compensate for the energy lost in one gait cycle of uphill walking [31]. However, this should not be misinterpreted as, in practise, a finite accumulator capacity and an upper limit on system pressure will limit the distance that can be walked uphill before the accumulator requires recharging or push-off is lost. In other words, only short periods of uphill walking could be powered by the energy stored during level walking. Having said this, when the accumulator has discharged the system could simply revert to operating like a traditional prosthesis, where the parallel spring provides ankle compliance but push-off is lost.

If improvements in design led to the total losses over the gait cycle being halved, then the increase in accumulator energy over the gait cycle would be doubled to $+44 \%$ of normal push-off. Note that the apparent inverse proportionality is coincidental. The gearbox and VDA losses during swing are $35 \%$ of the total and it may be possible to improve energy efficiency by disconnecting the device during swing and relying on an additional restoring spring to dorsiflex the foot for ground clearance. Furthermore, it may be possible to design a bespoke miniature VDA with lower losses than assumed here (i.e. lower than published by McCandlish and Dorey [30]).

In healthy gait the knee does more eccentric work than it does concentric work [11], [12]. Therefore, for transfemoral amputees, a second actuator (gearbox and VDA) and spring could be used to provide a normal knee torque profile while harvesting the excess eccentric work. This could be used to further recharge a common accumulator and then used for ankle push-off. The net work absorbed at the knee could be of the order of $10 \mathrm{~J}$ of which we might conservatively expect $5 \mathrm{~J}$ to be stored (an additional $40 \%$ of normal push-off). 


\section{B. Practical design issues}

In this section we present estimates of the weights of the main components in an attempt to gauge the practicality of the proposed design. But it should be emphasised that, at this stage, only rough estimates are possible. DESIGN_1 utilises a VDA with a maximum displacement of just $0.256 \mathrm{cc} / \mathrm{rev}$ which is smaller than anything we could find that is commercially available. The smallest commercial device we could find was $0.5 \mathrm{cc} / \mathrm{rev}$ with a mass of $0.8 \mathrm{~kg}$ (Eaton Aerospace). However, as both mass and displacement depend on dimensions cubed, with advanced miniaturisation one would expect mass to be proportional to displacement. For the Eaton Aerospace range, there is indeed a linear relationship but it does not go through the origin indicating a lower limit to the current ability to achieve miniaturisation. If future improvements in miniaturisation lead to a genuinely proportional relationship (i.e. a linear relationship going through the origin) then the mass of a $0.256 \mathrm{cc} / \mathrm{rev}$ VDA would be $0.07 \mathrm{~kg}$. Whilst we don't suggest that this is realistic, it does demonstrate that advanced miniaturisation could achieve substantial weight reductions. Here we make the pragmatic assumption that $0.3 \mathrm{~kg}$ is possible if some improvements in miniaturisation can be achieved.

DESIGN_1 utilises a gearbox ratio of 26 with a maximum input torque of $22 \mathrm{Nm}$ (both rounded up). The mass of an offthe-shelf planetary gearbox for this specification would be of the order of $0.5 \mathrm{~kg}$ (Reliance Precision). This is based on standard components using common engineering materials such as stainless steel and aluminium alloy. Using alternative materials, such as titanium alloys and composites, it should be possible to design a bespoke gearbox built into a prosthetic ankle that is significantly lighter than this, perhaps as low as $0.3 \mathrm{~kg}$.

In this report we have assumed an accumulator capacity of $250 \mathrm{cc}$. If a standard off-the-shelf metallic accumulator was sourced, this would have a mass of approximately $1 \mathrm{~kg}$. However, composite construction would reduce this by around $75 \%$ to give a mass of $0.25 \mathrm{~kg}$ (http://www.ctgltd.com/product/hydraulic-accumulators).

Furthermore, the pylon and accumulator could share structural elements, reducing the increase in mass associated with the accumulator.

Finally, the mass of the oil used needs to be estimated. Assuming a pre-charge pressure of $90 \mathrm{bar}$ and a maximum pressure of 200bar, the maximum oil volume in the accumulator would be of the order of $110 \mathrm{cc}$. If we add $50 \mathrm{cc}$ for the oil in the rest of the system, we would have $160 \mathrm{cc}$ with an approximate mass of $0.15 \mathrm{~kg}$.

We assume that the combined weight of the parallel spring, prosthetic foot, pylon and other componentry would be similar to a traditional trans-tibial prosthesis (circa 1.6kg) [32]-[34].

In summary, using the arguments above, we believe it may be possible to achieve the following masses but we emphasise that these are only rough estimates:

VDA $-0.3 \mathrm{~kg}$

Gearbox $-0.3 \mathrm{~kg}$

Accumulator $-0.25 \mathrm{~kg}$

Hydraulic oil $-0.15 \mathrm{~kg}$
Parallel spring, prosthetic foot, other componentry $-1.6 \mathrm{~kg}$

\section{TOTAL - 2.6kg}

The mass of the BiOM powered prosthesis is $2.3 \mathrm{~kg}$, which suggests that the design approach proposed here would be worthy of further investigation if a suitable VDA could be sourced. However, the design of a miniature, low-losses, lightweight VDA (most likely a variable displacement radial or axial piston unit) represents a major challenge in its own right.

\section{Limitations}

As discussed in the methods section, although the input data for level walking is optimistic with respect to the amount of eccentric work done, it is nevertheless within the range of data seen in healthy gait. We chose to use this optimistic data because there is an argument that amputees would benefit from walking in a manner that provides good push-off if it helped reduce their overall cost of locomotion. Therefore, the feasibility of a hydraulic VDA based prosthetic ankle will depend heavily on whether the eccentric ankle work needed prior to push-off is justified by the benefits of a more normal push-off. This is a question that can only be answered through in-vivo testing with amputees. To put this in context, if the eccentric work prior to push-off was halved, then the accumulator would discharge at the rate of 7.7 joules per gait cycle (63\% of normal push-off), which would largely negate the benefits of the system.

Our simulation models involve simplifications and assumptions that have been described in the Methods section. The errors introduced by most of these can only be accurately determined via physical prototyping and testing.

We have presented estimates of the weights of the main components in an attempt to gauge the practicality of the proposed design. But, at this stage, only rough estimates are possible.

\section{CONCLUSION}

The simulation results for DESIGN_1 are promising and suggest that, despite the significant losses involved, a hydraulic VDA based prosthetic ankle could improve amputee gait by restoring normal push-off. However, there are many unanswered questions, which means that our confidence in this conclusion is not high. For this approach to be a success, a new miniature, low-losses, lightweight VDA would be required that is half the displacement of the smallest commercially available device we could find. When such a device becomes available, we believe it would then be appropriate to move on to physical prototyping so that the invivo effect on amputee walking could be assessed.

\section{ACKNOWLEDGMENTS}

This work was funded by the Engineering and Physical Sciences Research Council (Swindon, United Kingdom) (grant $\mathrm{EP} / \mathrm{K} 019759 / 1)$. We also appreciate the comments of two anonymous reviewers which improved the final manuscript. 


\section{REFERENCES}

[1] L. J. Mengelkoch, J. T. Kahle, and M. J. Highsmith, "Energy costs \& performance of transtibial amputees \& non-amputees during walking \& running," Int. J. Sports Med., vol. 35, no. 14, pp. 1223-1228, Aug. 2014.

[2] T. Schmalz, S. Blumentritt, and R. Jarasch, "Energy expenditure and biomechanical characteristics of lower limb amputee gait: the influence of prosthetic alignment and different prosthetic components.," Gait Posture, vol. 16, no. 3, pp. 255-63, Dec. 2002.

[3] D. H. Nielsen, D. G. Shurr, J. C. Golden, and K. Meier, "Comparison of energy cost and gait efficiency during ambulation in below-knee amputees using different prosthetic feet - a preliminary report," JPO J. Prosthetics Orthot., vol. 1, no. 1, pp. 24-31, 1988.

[4] J. J. Genin, G. J. Bastien, B. Franck, C. Detrembleur, and P. Willems, "Effect of speed on the energy cost of walking in unilateral traumatic lower limb amputees.," Eur. J. Appl. Physiol., vol. 103, no. 6, pp. 65563, Aug. 2008

[5] C. Hofstad, H. Linde, J. Limbeek, and K. Postema, "Prescription of prosthetic ankle-foot mechanisms after lower limb amputation.," Cochrane database Syst. Rev., no. 1, p. CD003978, Jan. 2004.

[6] J. Gardiner, A. Z. Bari, D. Howard, and L. Kenney, "Energy storage and return prosthetic feet have only marginally improved trans-tibial amputee gait efficiency compared to that with solid ankle cushioned heel feet," J. Rehabil. Res. Dev., vol. 53, no. 6, pp. 1133-1138, 2016.

[7] S. Lipfert, M. Günther, D. Renjewski, and A. Seyfarth, "Impulsive ankle push-off powers leg swing in human walking," J. Exp. Biol., vol. 217, pp. 1218-1228, Dec. 2013.

[8] K. E. Zelik, T.-W. P. Huang, P. G. Adamczyk, and A. D. Kuo, "The role of series ankle elasticity in bipedal walking.," J. Theor. Biol., vol. 346, pp. 75-85, Apr. 2014.

[9] A. Ruina, J. E. A. Bertram, and M. Srinivasan, "A collisional model of the energetic cost of support work qualitatively explains leg sequencing in walking and galloping, pseudo-elastic leg behavior in running and the walk-to-run transition,” J. Theor. Biol., vol. 237, no. 2, pp. 170-192, 2005.

[10] K. Postema, H. Hermens, J. de Vries, H. Koopman, and W. Eisma, "Energy storage and release of prosthetic feet Part 1: Biomechanical analysis related to user benefits," Prosthet. Orthot. Int., vol. 21, no. 1, pp. 17-27, 1997.

[11] A. D. Segal, M. S. Orendurff, G. K. Klute, M. L. McDowell, J. a. Pecoraro, J. Shofer, and J. M. Czerniecki, "Kinematic and kinetic comparisons of transfemoral amputee gait using C-Leg and Mauch SNS prosthetic knees," J. Rehabil. Res. Dev., vol. 43, no. 7, p. 857, 2006.

[12] A. E. Ferris, J. M. Aldridge, C. A. Rábago, and J. M. Wilken, "Evaluation of a powered ankle-foot prosthetic system during walking.," Arch. Phys. Med. Rehabil., vol. 93, no. 11, pp. 1911-1918, Nov. 2012.

[13] R. A. Weinert-Aplin, D. Howard, M. Twiste, H. L. Jarvis, A. N. Bennett, and R. J. Baker, "Energy flow analysis of amputee walking shows a proximally-directed transfer of energy in intact limbs, compared to a distally-directed transfer in prosthetic limbs at push-off.," Med. Eng. Phys., vol. 39, pp. 73-82, 2017.

[14] R. J. Williams, A. H. Hansen, and S. a Gard, "Prosthetic ankle-foot mechanism capable of automatic adaptation to the walking surface.," $J$. Biomech. Eng., vol. 131, no. 3, p. 35002, Mar. 2009.

[15] S. H. Collins and A. D. Kuo, "Recycling energy to restore impaired ankle function during human walking.," PLoS One, vol. 5, no. 2, p. e9307, Jan. 2010.

[16] A. D. Segal, K. E. Zelik, G. K. Klute, D. C. Morgenroth, M. E. Hahn, M. S. Orendurff, P. G. Adamczyk, S. H. Collins, A. D. Kuo, and J. M. Czerniecki, "The effects of a controlled energy storage and return prototype prosthetic foot on transtibial amputee ambulation.," Hum. Mov. Sci., vol. 31, no. 4, pp. 918-31, Aug. 2012.

[17] S. K. Au, J. Weber, and H. Herr, "Biomechanical Design of a Powered Ankle-Foot Prosthesis," in Rehabilitation Robotics, 2007. ICORR 2007. IEEE 10th International Conference on, 2007, pp. 298-303.

[18] H. Herr, D. Paluska, and P. Dilworth, "Artificial human limbs and joints employing actuators, springs, and variable-damper elements," US 2006/0249315 A1, 2006.

[19] H. M. Herr and A. M. Grabowski, "Bionic ankle-foot prosthesis normalizes walking gait for persons with leg amputation.," Proc. Biol. Sci., vol. 279, no. 1728, pp. 457-64, Feb. 2012.

[20] A. J. van den Bogert, S. Samorezov, B. L. Davis, and W. a Smith, "Modeling and optimal control of an energy-storing prosthetic knee.," $J$. Biomech. Eng., vol. 134, no. 5, p. 51007, May 2012.
[21] E. Iversen, H. Sears, A. Dyck, S. Kunz, J. Jacobs, J. Linder, and P. Strazdins, "Device and system for prosthetic knees and ankles," US 2006/0235544 A1, 2006.

[22] J. Loverich, D. Kraige, J. Frank, and R. Geiger, "Apparatuses and methods for harvesting energy from prosthetic limbs," WO 2013/177589 A1, 2013 .

[23] D. Fairbanks, A. Zoss, V. Pillai, M. Schwartz, N. Harding, M. Rosa, B. Gilbert, A. Lambrecht, S. Kruse, and H. Kazerooni, "Semi-actuated transfemoral prosthetic knee," US 2010/0023133 A1, 2010.

[24] R. L. Waters, J. Perry, D. Antonelli, and H. Hislop, "Energy cost of walking of amputees: the influence of level of amputation," J Bone Jt. Surg Am, vol. 58, no. 1, pp. 42-46, Jan. 1976.

[25] P. A. Macfarlane, D. H. Nielsen, D. G. Shurr, K. G. Meier, R. Clark, J. Kerns, M. Moreno, and B. Ryan, "Transfemoral Amputee Physiological Requirements: Comparisons Between SACH Foot Walking and FlexFoot Walking," JPO J. Prosthetics Orthot., vol. 9, no. 4, 1997.

[26] A. Z. Bari, "An efficient energy storage and return prosthesis," $P h D$ thesis, Univ. Salford, 2013.

[27] A. H. Hansen, D. S. Childress, S. C. Miff, S. a Gard, and K. P. Mesplay, "The human ankle during walking: implications for design of biomimetic ankle prostheses.," J. Biomech., vol. 37, no. 10, pp. 146774 , Oct. 2004

[28] C. V. Jutte and S. Kota, "Design of Nonlinear Springs for Prescribed Load-Displacement Functions," J. Mech. Des., vol. 130, no. 8, p. 81403, 2008.

[29] C. V. Jutte and S. Kota, "Design of Single, Multiple, and Scaled Nonlinear Springs for Prescribed Nonlinear Responses," J. Mech. Des., vol. 132 , no. 1 , p. 11003,2010

[30] D. McCandlish and R. E. Dorey, "The Mathematical Modelling of Hydrostatic Pumps and Motors," Proc. Inst. Mech. Eng. Part B J. Eng. Manuf., vol. 198, no. 3, pp. 165-174, 1984.

[31] J. D. Gardiner, L. P. J. Kenney, and D. Howard, "Performance of optimised prosthetic ankle designs that are based on a hydraulic variable displacement actuator (VDA)," Intern. Report, Univ. Salford, 2015.

[32] R. S. Gailey, M. S. Nash, T. a Atchley, R. M. Zilmer, G. R. MolineLittle, N. Morris-Cresswell, and L. I. Siebert, "The effects of prosthesis mass on metabolic cost of ambulation in non-vascular trans-tibial amputees.," Prosthet. Orthot. Int., vol. 21, no. 1, pp. 9-16, Apr. 1997.

[33] S. J. Lin-Chan, D. H. Nielsen, H. J. Yack, M. J. Hsu, and D. G. Shurr, "The Effects of Added Prosthetic Mass on Physiologic Responses and Stride Frequency during Multiple Speeds of Walking in Persons with Transtibial Amputation," Arch. Phys. Med. Rehabil., vol. 84, no. 12, pp. 1865-1871, 2003.

[34] J. F. Lehmann, R. Price, R. Okumura, K. Questad, B. J. De Lateur, and A. Négretot, "Mass and mass distribution of below-knee prostheses: Effect on gait efficacy and self-selected walking speed," Arch. Phys. Med. Rehabil., vol. 79, no. 2, pp. 162-168, 1998. 